\title{
Trigger Detectors for the LHCb Muon System VCI 2001
}

\author{
W. Riegler ${ }^{a}$ for the LHCb muon collaboration \\ ${ }^{a} C E R N$, Geneva, Switzerland
}

\begin{abstract}
The muon detector of $\mathrm{LHCb}$ consists of five tracking stations which are placed along the beam axis and separated by iron filters. A muon trigger requires the coincidence of hits in all stations which demands a time resolution better than $3 \mathrm{~ns}$ and an efficiency of $>99 \%$ for each station. Resistive Plate Chambers and Multi Wire Proportional Chambers with wire and cathode readout are used for this purpose. The general signal formation and timing characteristics of RPCs and MWPCs for this application are discussed and compared. Test results of chamber prototypes are presented.
\end{abstract}

\section{Introduction}

A schematic view of the LHCb experiment [1] is shown in Figure 1. The first muon station (M1) is positioned around $12 \mathrm{~m}$ from the interaction point in front of the calorimeter. The other stations (M2-M5) behind the calorimeter are separated by $80 \mathrm{~cm}$ iron filters and the granularity increases projectively. The total detector area is $435 \mathrm{~m}^{2}$, the spatial resolution requirement ranges from $0.6 \mathrm{~cm}$ in the inner regions to a $30 \mathrm{~cm}$ in the outer regions. A muon trigger is given by a coincidence of all five muon stations within the bunch crossing time of $25 \mathrm{~ns}$ in a certain spatial window that selects the muon momentum. Therefore each muon station is required to have $>99 \%$ efficiency in a $20 \mathrm{~ns}$ time window.

\section{Trigger Detectors}

Resistive Plate Chambers (RPCs) [2] will be used in regions with rates $<1 \mathrm{kHz} / \mathrm{cm}^{2}$ and Multi Wire Proportional Chambers (MWPCs) [3] will be used all other regions with rates up $100 \mathrm{kHz} / \mathrm{cm}^{2}$. A schematic view of the detectors is shown in Figure 2. 


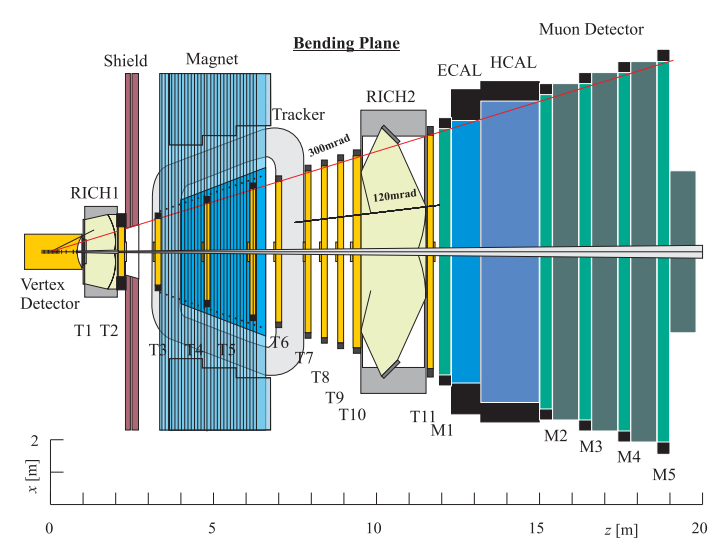

Fig. 1. The LHCb Experiment. The five muon stations are labelled M1-M5.

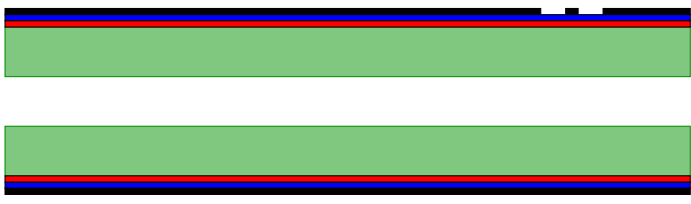

00000000

Fig. 2. Schematic view of an RPC and a MWPC. Two RPCs or four MWPCs will form one muon station.

The RPCs use $2 \mathrm{~mm}$ Bakelite with a volume resistivity of $9 \times 10^{9} \Omega \mathrm{cm}$. The $2 \mathrm{~mm}$ gas gap is filled with $\mathrm{C}_{2} \mathrm{~F}_{4} \mathrm{H}_{2} / \mathrm{i}-\mathrm{C}_{4} \mathrm{H}_{10} / \mathrm{SF}_{6} 95 / 4 / 1$ [4]. The working point is around $10 \mathrm{kV}$ giving an electric field of $50 \mathrm{kV} / \mathrm{cm}$ in the gas gap.

The MWPCs use a $5 \mathrm{~mm}$ gas gap, $1.5 \mathrm{~mm}$ wire pitch and $30 \mu \mathrm{m}$ wire. The gas mixture $\mathrm{Ar} / \mathrm{CO}_{2} / \mathrm{CF}_{4} 40 / 50 / 10$ is operated at a wire voltage of $3.15 \mathrm{kV}$. At this voltage the field on the cathode surface is about $8 \mathrm{kV} / \mathrm{cm}$, so one has to avoid sharp edges on the cathode surface.

\section{Performance Comparison}

To compare the detector characteristics a detailed simulation of the RPCs and MWPCs was performed using GARFIELD [5], MAGBOLTZ [6] and HEED $[7]$.

For a $10 \mathrm{GeV}$ muon, HEED predicts on average 20.2 clusters for the $2 \mathrm{~mm}$ 
RPC gap and 21.4 clusters for the $5 \mathrm{~mm}$ MWPC gap. The larger ionization for the RPC is due to the fact that the RPC gas is about 2.2 times heavier than the MWPC gas.

For the drift velocity we assume $100 \mu \mathrm{m} / \mathrm{ns}$ for the RPC and MAGBOLTZ predicts $90 \mu \mathrm{m} / \mathrm{ns}$ for the MWPC gas $(8 \mathrm{kV} / \mathrm{cm})$. The RPCs work in limited space charge mode which is possible due to the strong streamer suppression provided by the $\mathrm{SF}_{6}$. The space charge effect was not included in the simulation and an effective Townsend coefficient of $95 \mathrm{~cm}^{-1}$ was assumed to reproduce the measured charge. The MWPCs are operated in proportional mode and measurements show a gain of $10^{5}$ for a voltage of $3.15 \mathrm{kV}$.

The total avalanche charge is about $37 \mathrm{pC}$ for the $\mathrm{RPC}$ and $0.74 \mathrm{pC}$ for the MWPCs. The signal shapes differ significantly for the two detectors. In the RPCs only the electrons induce a significant signal, accounting for $1.5 \mathrm{pC}$ of the total charge. Therefore the signal is very short, i.e. around $10 \mathrm{~ns}$ for the above geometry. The ions take about $2 \mu$ s to move back to the Bakelite surface. The wire chamber signal contains a short electron component and a long ion component lasting for about $20 \mu \mathrm{s}$ with a $1 /\left(t+t_{0}\right)$ shape and $t_{0} \approx 3 \mathrm{~ns}$. A dedicated tail cancellation network in the frontend electronics is used to shorten the signal which results in an average pulse width of $\approx 50 \mathrm{~ns}$.

After simulation of the avalanche we find the induced charge spectrum which is 'exponential' for the RPC and 'Landau like' for the wire chamber. This difference, despite the fact that there are equal numbers of primary clusters in both detectors, is due to the fact that in the RPC only the first clusters in the gap can develop a full avalanche while for the MWPCs every single electron can develop a full avalanche close to the wire.

To find the intrinsic time resolution of the detectors we can investigate the time resolution for a given threshold in $\mathrm{fC}$, i.e. we integrate the signal until it crosses a certain threshold. The simulation shows a time resolution of $\approx 1.2 \mathrm{~ns}$ for the RPC which is almost independent of the threshold up to $100 \mathrm{fC}$. For the MWPC the resolution decreases rapidly from 3 ns at $0.1 \mathrm{fC}$ to $6 \mathrm{~ns}$ at $10 \mathrm{fC}$ threshold. The difference comes from the fact that for the RPC the charge is induced within a very short time while in the wire chamber the drift of the electrons to the wire accounts for about $2.5 \mathrm{~ns}$ and in addition the slowly moving ions take a long time to induce the charge. We note here that for high threshold the RPCs become inefficient due to small signals staying below the threshold, while the MWPCs become inefficient due to decreased time resolution and resulting hits outside the $20 \mathrm{~ns}$ time window.

The choice of the frontend electronics bandwidth is finally determined by the time resolution requirement. For the RPCs the simulation shows time resolutions of $1.3,1.5$ and $1.8 \mathrm{~ns}$ for amplifier peaking times of 3,10 and $15 \mathrm{~ns}$, 
fairly independent of the threshold. For the MWPCs we find time resolutions of 5.6, 5 and $5.2 \mathrm{~ns}$ for peaking times 3, 10 and $15 \mathrm{~ns}$ and a threshold of 8 primary electrons. The resolution decreases rapidly for higher threshold. The optimum peaking time is around $8 \mathrm{~ns}$, faster amplifiers do not collect enough charge while for slower amplifiers the time slewing effect starts to dominate. Measurements performed with 3 ns peaking time for the RPCs and 10 ns peaking time for the MWPCs compare very well with the simulated values. The study shows that it is feasible to use the same electronics with a peaking time of $8 \mathrm{~ns}$ for the RPCs and MWPCs.

Crosstalk issues are discussed in detail in [8]. Figure 3 finally shows the measured performance of a double RPC and a double MWPC. The performance fulfils the requirements for the LHCb muon system. For redundancy reasons 4 MWPCs will form one muon station.

\section{Conclusions}

Resistive Plate Chambers and Wire Chambers for the LHCb muon system were studied in detail. Apart from space charge effects in the RPCs the detectors can be simulated very well and the characteristics are well understood. Two RPC layers or $4 \mathrm{MWPC}$ layers fulfil the requirements for the LHCb muon system.

\section{References}

[1] LHCb Technical Proposal - LHCb Collaboration. CERN-LHCC-98-004 (1998).

[2] M. Adinolfi et. al., Proposal for the RPC muon detector of LHCb, LHCb note, LHCb-2000-053, CERN 2000.

[3] B. Botchine et. al., Wire pad chambers for the LHCb muon system, LHCb internal note, LHCb-2000-003, March 2000

[4] R. Camarri et. al., Streamer suppression with SF6 in RPCs operated in avalanche mode, NIM A414 (1998) 317-324

[5] Rob Veenhof, GARFIELD, a drift chamber simulation program, Version 7.02, CERN

[6] S. Biagi, MAGBOLTZ, program to compute gas transport parameters, Version 2.2, CERN

[7] Igor Smirnov, HEED, program to compute energy loss of fast particles in gases, Version 1.01, CERN 

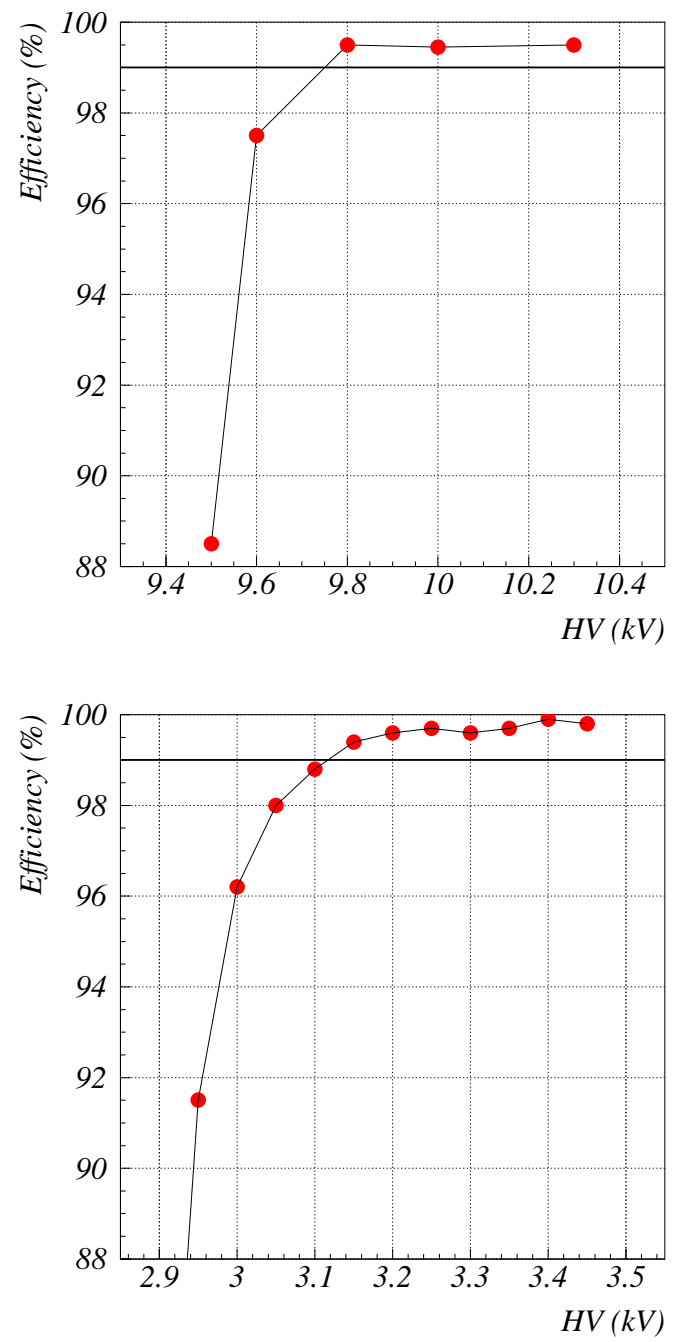

Fig. 3. Measured efficiency of a double RPC and a double MWPC for a $20 \mathrm{~ns}$ time window.

[8] W. Riegler, D. Burgarth, Signal Propagation, Termination, Crosstalk and Losses in Resistive Plate Chambers. CERN preprint CERN-EP/2001-014 Feb. 2001. 